\title{
Tooth loss in treated periodontitis patients responsible for their supportive care arrangements
}

\author{
W. Keung Leung, Daniel K.C. Ng, Lijian Jin and Esmonde F. Corbet \\ Faculty of Dentistry, The University of Hong Kong, Hong Kong SAR, China
}

Running head: Periodontal outcomes without regular SPC

Corresponding author:

W. Keung Leung

Faculty of Dentistry

The University of Hong Kong

Room 3B39, Prince Philip Dental Hospital, 34 Hospital Road

Hong Kong SAR

China

Tel: $\quad 852-2859-0417$

Fax: $\quad 852-2858-7874$

Email: ewkleung@hkucc.hku.hk

Date of submission: September 15, 2005

Date of revision: December 17, 2005 


\begin{abstract}
Aim: To identify risk indicators associated with tooth loss and periodontitis in treated patients responsible for arranging supportive periodontal care.

Materials and Methods: Ninety seven Chinese subjects (34-77 years) who showed favourable responses to periodontal therapy provided in a teaching hospital 5-12 years previously were recalled. They were advised to seek regular supportive periodontal care on discharge. Background information, general health status, smoking, oral hygiene habits, follow-up dental care, tooth loss, and periodontal parameters were investigated. Multiple regression analysis was performed.

Results: Two hundred fifty six teeth had been lost; 195 due to self-reported periodontal reasons. Up to $26.8 \%$ sites were with pockets $\geq 6 \mathrm{~mm}$. Positive correlations were found between total/periodontal tooth loss and i) smoking-packyears, ii) time spent on oral hygiene, iii) years since therapy's conclusion, iv) age; and negative correlations with v) interdental brush use, and vi) education levels. Tooth loss by arch was correlated with removable partial denture wearing in that arch. Percentage sites with pockets $\geq 6 \mathrm{~mm}$ was significantly negatively correlated with percentage sites without bleeding on probing.
\end{abstract}

Conclusions: Smokers, more elderly patients, removable partial denture wearers, patients with lower education levels or not using interdental brushes ought to be targeted for clinic-based supportive periodontal care.

Key words: Home care; periodontal diseases; smoking; supportive periodontal care; tooth loss 
Chronic periodontitis is an inflammatory disease of the human periodontium caused by opportunistic infection by dental plaque pathogens in susceptible individuals (Kornman et al. 1997). Long-term carefully managed plaque control programme in one dental office has shown that the disease may be largely prevented over a 30 -year period (Axelsson et al. 2004). Non-surgical therapy (van der Weijden \& Timmerman 2002) and/or surgical therapy (Heitz-Mayfield et al. 2002) are effective treatments and it is generally accepted that active treatment should be followed by a regularly delivered supportive therapeutic or care programme. This long-term care for treated periodontitis patients aims at preventing or minimizing oral disease recurrence, preventing or reducing tooth loss, and allowing the diagnosis and treatment of new disease. This long-term care has been variously termed supportive periodontal therapy (American Academy of Periodontology [AAP] 1998), supportive periodontal care (SPC) (Heasman et al. 2002), or periodontal maintenance (American Academy of Periodontology [AAP] - Cohen 2003). As a regime to maintain periodontal health, SPC when properly, regularly and frequently carried out, is successful (Axelsson \& Lindhe 1981). Persistence of periodontal destruction and recurrent periodontitis are not uncommon, even for subjects undergoing the standard regimen of 3-monthly regular SPC (Fleming et al. 1996). In recognizing this, and that some previously treated patients may not require such careful maintenance, professional organisations, such as the AAP, have recommended guidelines for supportive periodontal therapy/periodontal maintenance, stressing that supportive care should be individualized (AAP 1998, Cohen 2003).

Studies on factors related to unfavourable long-term treatment outcomes have included: smoking, periodontal disease experience, irregular dental visits, gingival inflammation, bleeding on probing, systemic medical conditions and elevated 
subgingival periodontopathogen levels (Ainamo \& Ainamo 1996, Rams et al. 1996, Bergstrom et al. 2000, Molloy et al. 2004, Rahardjo et al. 2005, Schätzle et al. 2004). Despite much being known about periodontal diseases and their treatment outcomes (Heitz-Mayfield 2005), disease activity rates might be so low as to render data interpretation for prospective studies of disease history or randomized clinical trials of disease intervention very difficult (Antczak-Bouckoms 1994). Thus, retrospective studies of treatment outcomes, including tooth loss, appear to have a place.

Not every patient treated for periodontitis in a teaching hospital environment can be offered SPC according to generally recommended recall systems. Hence it would be beneficial to be able to identify those treated periodontitis patients whose long-term treatment outcome would be adversely affected by a lack of regular SPC. On the basis of one study of periodontal maintenance patients of a University Clinic it was suggested that better knowledge of risk indicators may lead to more efficient management efforts during periodontal maintenance (Tonetti et al. 1998). The goal of the current retrospective study was thus to identify possible risk indicators and other variables identified from clinical examination and questionnaire interview associated with new or recurrent periodontitis in the form of tooth loss experience and periodontal pockets at recall in a group of previously successfully treated periodontal patients, who were responsible for their supportive care arrangements. The recruits had not been subject to regular SPC within the clinic in which the previously successful periodontal therapy had been performed. The information gained would guide the selection of patients who had deteriorated most due to a lack of SPC in the Clinic and who thus might benefit most from regularly delivered SPC in the University Periodontology clinic with a scarcity of resources for delivering SPC to all periodontitis patients treated within that clinic. 


\section{Material and Methods}

\section{Subjects}

A list of patients who had either what was then categorised as rapidly progressive or adult periodontitis, who had undergone supervised periodontal therapy by final year undergraduate dental students at least 5 years before the commencement date of this study, and who had been subsequently discharged with instructions on the need for them to make their own arrangements for SPC, was generated from the Periodontology Clinic records $(n=439)$. All patient cases were presentation cases nominated by graduating dental students from each student's patient pool and used as part of the final professional examination at the Faculty of Dentistry, The University of Hong Kong. All non-surgical periodontal therapy had been provided by the students and staff dental hygienists. Periodontal surgery when indicated was also provided by the students under supervision or by postgraduate students or staff of the Periodontology Clinic. The dental student concerned also provided or arranged for the provision of comprehensive dental treatment for the patient in addition to the periodontal therapy delivered. All patients received detailed explanations of the need for SPC to maintain the favourable periodontal treatment outcomes achieved, and verbal instructions to attend for regular SPC at general dentists were given to each patient not being offered SPC at the hospital before discharge from the Periodontology Clinic. In view of logistic and staffing reasons, regular SPC could not be delivered to all treated patients in the Periodontology Clinic, and the patients on the list of 439 had all been discharged to make their own SPC arrangements. The patient list was arranged in ascending order according to the hospital registration 
number. Every other subject $(\mathrm{n}=220)$ from the list was selected and screened against the criteria listed following for eligibility to this study.

All recruits for this study met the following inclusion criteria: (i) Chinese, (ii) no systemic problem at the time when treatment was delivered, (iii) females were not pregnant at the time of treatment, (iv) not undergoing orthodontic treatment, (v) clear documentation from the clinical records (chartings, clinical photographs, study casts and oral radiographs) showing favourable responses to periodontal treatment. The last entry of the student treatment record prior to discharge showed $\leq 20 \%$ of sites with bleeding on probing (BOP); no probing pocket depth (PPD) $\geq 6 \mathrm{~mm}$ and no more than $5 \%$ of probing sites with PPD $=5 \mathrm{~mm}$. Out of the 220 subjects selected, five were non-Chinese, ten had medical problems at the outset of treatment, two had incomplete dental records, 37 subjects had failed to demonstrate adequate treatment outcome (PPD $\geq 6 \mathrm{~mm}, \mathrm{PPD}=5 \mathrm{~mm}$ sites more than $5 \%$, or BOP sites $\geq 20 \%$ ), and 26 subjects resumed availing of periodontal care from the Periodontology Clinic subsequent to discharge. They were all excluded from the study. The remaining 140 previous patients were then invited by letter to attend for this study. One hundred and four of the subjects were successfully contacted and they agreed to take part in the present study. The research protocol was approved by the University of Hong Kong, Faculty of Dentistry's Ethics Committee, and written informed consent was obtained from all participants on attendance.

\section{Questionnaire and clinical examination}

Each recruit first completed a two-part questionnaire with a trained interviewer to record their demographical data, general health status, current oral hygiene habits, cigarette consumption patterns, denture wearing habits, general dental visit patterns 
since periodontal treatment had been completed in the dental hospital and an account of reasons for tooth loss, if any, since discharge. Subjects were encouraged to give, to their best of their recollection, the reasons for loss of each individual tooth subsequent to completion of periodontal treatment.

All subjects were examined by a single examiner (DKCN), blind to the responses to the questionnaire, who recorded number of teeth present and the fullmouth periodontal conditions including: Plaque presence/absence, BOP, and PPD using a Florida Probe ${ }^{\circledR}$. BOP was recorded as present or absent within 30 seconds after probing. Routine panoramic oral radiographs were taken. Arrangements for further periodontal treatment were made for each individual subject, dependent on the individual's clinical and dental care situation as appropriate.

\section{Data analysis}

Computer data analysis was carried out using the statistics software StatView ${ }^{\circledR}$ (Abacus Concepts Inc., Berkeley, CA). Standard descriptive statistics were used to summarize all variables studied. Linear multiple regression analysis was performed to determine if there was any correlation between: i) tooth loss after previous periodontal therapy, ii) current proportion of sites with $\mathrm{PPD} \geq 6 \mathrm{~mm}$, and 17 independent variables of the patients. These variables were: a) categorical data - sex, general health status, current denture wearing, education levels, five oral hygiene variables (including usage of various interdental devices, and frequency of daily tooth brushing); b) continuous data - age, smoking in pack-years, years elapsed since the end of previous periodontal therapy, self-reported time spent for daily oral hygiene, percentage sites with plaque on recall examination, percentage sites without BOP $(\mathrm{BOP}=0)$ on recall examination, percentage of teeth with PPD $\geq 6 \mathrm{~mm}$ at beginning 
of previous periodontal therapy and number of remaining teeth at the end of the previous periodontal therapy. The analysis method recommended by Beck (1994) was followed.

\section{Results}

\section{Patient demography and general profiles}

Ninety-seven (59 female, $60.8 \%$ ) out of the 104 recruited previously treated patients attended for examination. Their age range at completion of previous periodontal therapy was 25-67 years (mean $40.6 \pm 9.2$ years), and at recall was 34-77 years (mean $50.2 \pm 9.5$ years). All had completed treatment $5-12$ years (mean $8.9 \pm 2.3$ years) ago. Six of the previously treated patients $(6.2 \%)$ had received none/pre-school education, $29(29.9 \%), 53(54.6 \%), 3(3.1 \%)$ and $6(6.2 \%)$ had received primary school, secondary schools, tertiary (non-degree), or University degree or above education, respectively.

Eleven $(11.3 \%)$ of the previously treated patients were current smokers with self-reported cumulative consumption of 0.5-56.9 pack-years (mean 23.0 \pm 20.6 pack years). Self-reported medical health condition evaluation revealed that $20(20.6 \%)$ participants had systemic disease(s) on recall. Some of these previously treated patients, who had been free from systemic disease at the time of their active treatment, were now suffering from various systemic conditions: diabetes $(n=6$, one with concurrent thyroid disease); hypertension $(n=6$, one with concurrent ischaemic heart disease); ischaemic heart disease $(n=2)$; thyroid disease $(n=2)$; hysterectomy and hormone replacement therapy $(n=1)$; benign ovary tumour $(n=1)$; asthma $(n=1)$; depression $(n=1)$. 
Patients who required removable partial denture(s) for aesthetics and/or functional reasons had been provided with the denture(s) as part of the comprehensive dental care upon completion of periodontal therapy. However, not every previously treated patient used the denture(s) delivered. Hence only those presenting for the study wearing removable partial dentures were considered to be denture wearers. Thirty-four $(35.1 \%)$ of the recruits wore removable partial dentures, with $8(8.2 \%)$ wearing lower dentures only, $11(11.3 \%)$ wearing upper dentures only, while 15 $(15.5 \%)$ wore both upper and lower dentures. Seventy-two (74.2\%) of the previously treated patients reported brushing their teeth twice a day, whereas only $8(8.2 \%)$ reported using single-tufted (end-tufted) toothbrushes. Thirty-five (36.1\%) of the recruits reported regularly using interdental brushes, 17 (17.5\%) reported regularly using dental floss and $56(57.7 \%)$ reported regularly using toothpicks. The reported time devoted to the daily oral hygiene practice ranged from 1-30 minutes (mean $5.8 \pm$ $4.4 \mathrm{~min})$.

Five $(5.2 \%)$ previously treated patients had not sought any dental treatment since their discharge from the dental hospital. Seventy-four $(76.3 \%)$ had visited dentists at intervals greater than one year for scaling, the remaining 18 (18.6\%) participants reported regular dental visits for scaling at intervals of less than twelve months, but none reported attendance patterns consistent with the advice on SPC intervals which they had received on discharge.

\section{Clinical parameters}

Of the 97 recruited subjects before previous periodontal treatment, 27 of them suffered at diagnosis from what then was known as rapidly progressive periodontitis and the rest $(n=70)$ suffered at diagnosis from chronic periodontitis. The relevant 
clinical parameters of the recruits before and at the conclusion of their previous periodontal therapy and at recall are shown in Table 1 . At the conclusion of their previous periodontal therapy, these 97 participants were found to have a total of 2522 standing teeth and approximately $2.0 \pm 0.8 \mathrm{~mm}(0.7-4.4 \mathrm{~mm})$ full mouth mean probing attachment loss (PAL), calculated from their clinical records. Ten subjects $(10.3 \%)$ were classified as minimal attachment loss (PAL $\leq 1.0 \mathrm{~mm}), 52(53.6 \%)$ as low (PAL $1.1-2.0 \mathrm{~mm}), 25(25.8 \%)$ as moderate (PAL $2.1-3.0 \mathrm{~mm}), 8(8.2 \%)$ as high (PAL $3.1-4.0 \mathrm{~mm}$ ), and $3(3.1 \%)$ as severe (PAL $\geq 4.1 \mathrm{~mm})$ PAL categories (Genco et al. 1999). No subject had active caries or non-healing periapical lesions, while the patients had a mean of $4.1 \pm 3.3(0-16)$ filled teeth and $0.3 \pm 0.7(0-4)$ root filled teeth. Eighty-five $(87.6 \%)$ of these patients had at least 21 remaining teeth at that time. The total number of remaining teeth by tooth type at the conclusion of the previous periodontal therapy is shown in Fig. 1. Two-hundred-fifty-six (10.2\%) teeth in these 97 subjects had been lost during the 5-12 year post-treatment period (maxillary, $\mathrm{n}=143$; mandibular, $\mathrm{n}=113)$, of which $195(7.7 \%$ of total) or $76.2 \%$ of teeth lost (195/256) were reported to have been lost due to periodontal reasons (maxillary, $\mathrm{n}=108$; mandibular, $\mathrm{n}=87$ ). The number of remaining teeth lost for all reasons and due to periodontal reasons is shown in Fig. 2 for each tooth type. Different tooth types experienced different likelihoods of loss for periodontal reasons. More than $10 \%$ each of upper first, second and third molars, upper first premolars, lower first and second molars, and lower central incisors were lost during this posttreatment period (Fig. 2). Seventeen subjects (17.5\%) each had lost 5-14 teeth, accounting for $52.3 \%(134 / 256)$ of the total number of teeth lost for all reasons. Thirteen subjects (13.4\%) each had lost 5-13 teeth due to periodontal reasons and the tooth loss in this minority accounted for over half $52.8 \%(103 / 195)$ of the total 
number of teeth lost reported to be due to periodontal reasons. The profile of tooth loss for all subjects is shown in Fig. 3. On average, the annualized tooth loss was $0.25 \pm 0.26$ tooth per patient. Tooth loss due to periodontal reasons in smokers was 2.5 times that in non-smokers $-4.2 \pm 5.0$ vs $1.7 \pm 2.3$ teeth $(p=0.005)$.

On recall, 18 subjects had no PPD $>5.0 \mathrm{~mm}$. Four out of these patients were previously diagnosed to be suffering from what was then known as rapidly progressive periodontitis. Among the 18 subjects, 3 also had $\mathrm{Pl} \%$ and $\mathrm{BOP} \%<30 \%$, indicating that their periodontal health was reasonably well maintained. Six subjects out of the 18 had $\mathrm{BOP} \%<30 \%$ with $\mathrm{P} 1 \%$ ranging from $37-87 \%$; the rest had both high $\mathrm{BOP} \%$ (33-63\%) and $\mathrm{Pl} \%$ (50-94\%). Out of the 79 subjects with PPD > $5.0 \mathrm{~mm}$, only 6 had $\mathrm{P} 1 \%$ and $\mathrm{BOP} \%$ less than $30 \%$ and those subjects had $1-6$ sites $(0.6-4.2 \%)$ of PPD $>5.0 \mathrm{~mm}$. Eight subjects had $>10 \%$ sites $(10.1-26.8 \%)$ with $\mathrm{PPD} \geq 6.0 \mathrm{~mm}$. Of them, 2 suffered from rapidly progressive periodontitis at the time of the commencement of the previous periodontal therapy.

The 17 independent variables were subject to linear multiple regression analysis with i) further total tooth loss, ii) further tooth loss due to periodontal reasons and iii) percentage sites at re-examination with $\mathrm{PPD} \geq 6 \mathrm{~mm}$ as the dependent variables. Age, years elapsed since the end of previous periodontal treatment, smoking-pack-years, and self-reported daily oral hygiene practice duration were found to be related to both further total tooth loss and further tooth loss due to periodontal reasons (Tables $2 \& 3$ ). Usage of interdental brush and education level were negatively correlated with further total tooth loss and further tooth loss due to periodontal disease (Tables $2 \& 3$ ). Percentage of sites without BOP at reexamination was found to be negatively correlated with percentage of sites with PPD $\geq 6 \mathrm{~mm}$ (adjusted $R^{2}=0.282, F=38.28, p<0.0001$ ). The 17 independent variables 
were also subjected to linear multiple regression analysis with iv) further total tooth loss in the maxillary arch or the mandibular arch, and v) further tooth loss due to periodontal reasons in maxillary or mandibular arches. Generally, the same variables remained in the model for each arch separately for total tooth loss, except age and education level. When the tooth loss in the upper jaw was analysed, age was no longer part of the model. Education level was also no longer in the model for the upper jaw tooth loss due to periodontal reasons. Upper removable partial denture wearing was found to be positively correlated with total tooth loss (adjusted $R^{2}=$ 0.382, $F=10.87, p<0.0001$ ); and tooth loss due to periodontal reasons in the upper arch (adjusted $R^{2}=0.374, F=12.46, p<0.0001$ ). For tooth loss in the mandibular arch, only age remained in the model, and no other indicator except wearing of lower removable partial dentures was found to be positively correlated to total tooth loss (adjusted $R^{2}=0.186, F=11.96, p<0.0001$ ) and periodontal tooth loss in the lower $\operatorname{arch}\left(\right.$ adjusted $\left.R^{2}=0.172, F=10.96, p<0.0001\right)$.

\section{Discussion}

The present study investigated the periodontal conditions and tooth loss history of a group of periodontal patients 5-12 years post-therapy and investigated associations with a variety of variables gleaned from clinical examination and questionnaire interview. These periodontitis patients had been previously treated within a dental teaching hospital setting and had demonstrated favourable periodontal healing responses to periodontal therapy. Their clinical records showed a full-mouth mean of $2 \mathrm{~mm}$ PAL at the conclusion of their previous periodontal therapy. The treatment outcomes had been subject to internal scrutiny and audit. Hence it was assured that these patients had received active periodontal care delivered to an acceptable standard, 
albeit delivered by a range of student clinicians, with reasonably good initial periodontal treatment outcomes. Furthermore, these patients had all received comprehensive dental care in which all presenting dental disorders had been managed. Out of 220 patients selected on the basis of alternate selection 140 (64\%) were found to be eligible for inclusion. Of the 140 eligible patients 104 (74\%) were contactable. Of these 104 who were contacted 97 (93\%) were recruited into the study, a high recruitment rate. The demography of the finally recruited 97 subjects was similar to that of the non-selected individuals and the general population. For example, of the 219 non-selected subjects as retrieved from their records, 8.2\% had educational attainment of none/pre-school, $25.1 \%$ primary, $51.6 \%$ secondary, $11.9 \%$ tertiary (nondegree) and 3.2\% for University degree or above education, which were similar to the groupings of education levels for the 97 recruits and also the corresponding population data of $12.8 \%, 25.2 \%, 50.8 \%, 5.4 \%$, and $5.9 \%$ (Census and Statistic Department, Hong Kong, 1992).

All study subjects had been advised of the importance of regular SPC and had been instructed to seek for regular SPC from dental care providers outside the hospital, but none were found to have followed the advice given with respect to recommended intervals between visits. However a minority had received regular dental care, but with dental visits at far less than the recommended intervals. Many reports have shown that adequate compliance with SPC recommendations poses challenges for clinicians and patients world-wide, even in private periodontal offices: USA (Wilson et al. 1984, Wilson et al. 1993), Australia (Mendoza et al. 1991), Italy (Checchi et al. 1994), Greece (Demetriou et al. 1995), Brazil (Noaves et al. 1996), Japan (Ojima et al. 2001), and Iran (Soolari \& Rokn 2003), with one study in South America revealing cultural influences on compliance (Noaves et al. 1999). In general, world-wide, only 
about one-in-three treated periodontitis patients is fully compliant to periodontal recall regimens and high compliance to periodontal recall in practice has only been reported in a stable Norwegian population (Fardal et al. 2003). Nothing has been reported about compliance of Chinese patients to periodontal recalls, so recommendations about SPC resulting from this study may be modified by compliance issues. It has generally been shown that compliance positively affects the maintenance of favourable treatment outcomes (Checchi et al. 2002, König et al. 2002), even in smokers (Jansson \& Hagstrom 2002). If general dentists are given specific instructions about periodontal maintenance regimens, general dental practices can, in the short term at least, provide SPC resulting in favourable outcomes (Preshaw \& Heasman 2005).

Many of the patients recalled for this investigation did not have a regular dentist at the time of their discharge from the clinic, so it would not have been possible to instruct them. Regular SPC has been shown to make good for poorer treatment responses (Axtelius et al.1997) and to be effective in keeping tooth loss to a minimum in those with high susceptibility to periodontal disease (Rosling et al. 2001). Obviously the provision of regular SPC should be a component of the management of periodontitis, but not all treatment environments can offer life-long SPC to all treated periodontitis patients. The prime aim of this study was to identify possible risk factors, gleaned from questioning the patients and from their clinical examination at recall, associated with further tooth loss and/or relatively worse periodontal conditions at recall, reflected as greater proportions of sites with deep probing pocket depths, in these individuals who were not offered SPC at the Periodontology Clinic but who were responsible for arranging their own SPC. This information could guide in the identification of treated periodontitis patients who are likely not to maintain 
successful treatment outcomes, and who thus might benefit most were they to be targeted for regular SPC in the Periodontology Clinic, using systems for recall which have been shown to increase compliance (Anneken et al. 2001).

Tooth loss and percentage of sites with probing pocket depths $\geq 6 \mathrm{~mm}$ were used as indicators for periodontal disease progression and current periodontal status respectively. The PAL calculated from clinical records of the subjects at conclusion of the previous periodontal therapy, was not properly calibrated and was therefore considered less reliable than other clinical measures, and hence was not included in the model. Only those individuals who had demonstrated reasonably good periodontal treatment responses (Table 1) were included for study. To ensure the best possible management and documentation, only those previously treated patients whose clinical data and treatment responses had been through the scrutiny of a final professional dental degree examination and its attendant checks and balances were included. There was not a control group of similarly managed patients who were under the clinic based SPC regimens for comparison. The subjects in this study were previously affected by periodontal disease and were treated in an educational environment with heavy emphasis upon patient education about the natural history of periodontitis and the need for self-care. Thus, it is expected these patients would recall better than most people which teeth had been lost due to periodontal disease or for other reasons during the post-treatment period, so self-reporting was used to indicate teeth lost due to periodontitis. The percentage of pockets with probing depths $\geq 6 \mathrm{~mm}$ was chosen as another independent variable because, in addition to being an expression of current periodontal condition, this closely reflects periodontal disease experience (Claffey \& Egelberg 1995) and risk for progression (Renvert \& Persson 2002). Since all participants at the end of previous periodontal therapy had responded well and did not 
have any deep pocket $\geq 6 \mathrm{~mm}$, findings of deep pockets would be part of the evidence for new or recurrent periodontitis.

Tooth loss was used as a measure of the end-stage of disease, similar to many previous studies of periodontally treated patients (Hirschfeld \& Wasserman 1978, McFall 1982, Becker et al. 1984, Goldman et al. 1986, Nabers et al. 1988, Wood et al. 1989, McLeod et al. 1997, Tonetti et al. 1998 \& 2000, Checchi et al. 2002, König et al. 2002). As in a similar teaching clinic-based study (Tonetti et al. 2000), the major reported reason for tooth loss in these treated periodontitis patients was periodontal. Periodontal disease has been shown to account for over one-third and up to nearly one-half of tooth extractions in adults of different age ranges in Hong Kong (Corbet \& Davies 1991), and on a subject level caries and periodontal disease variables were shown to be equally important predictors of tooth loss in elderly Chinese (Baelum et al. 1997). In the study subjects, three-quarters of the teeth lost subsequent to periodontal therapy were reportedly lost due to periodontal reasons. Total tooth loss in a patient population such as this, receiving continuing dental care in general dental practices, is the result of complex interactions (Worthington et al. 1999, Gilbert et al. 2002), especially for smokers. Therefore both total tooth loss and tooth loss due to periodontal reasons were each used separately as independent variables in the analyses. Due to the retrospective nature of this investigation and the nonstandardized nature of the initial records, levels of clinical attachment loss of the subjects were not followed. Tooth loss is a robust measure and accurate recordings of teeth present at the conclusion of active therapy existed. Panoramic oral radiographs, which it has been suggested are suitable for periodontal maintenance populations (Persson et al. 2003), were used to aid identify teeth present at recall. 
This study demonstrated negative correlation between percentage of sites without $\mathrm{BOP}$ at re-examination and percentage of sites with $\mathrm{PPD} \geq 6 \mathrm{~mm}$ which agrees with publications in the literature indicating that low BOP percentage in treated periodontitis patients signifies lower risk for recurrent periodontitis or disease progression (Joss et al. 1994).

As observed in pervious studies (Hirschfeld \& Wasserman 1978, McFall 1982, Goldman et al. 1986), a minority of subjects in this study was responsible for a large proportion of tooth loss (Fig. 3). The findings on further tooth loss reported due to periodontal disease in the present study were to some extent similar to tooth loss in the report of regularly maintained periodontal patients by Hirschfeld and Wasserman (1978). These similarities included bilateral symmetry of tooth loss, upper teeth were lost more frequently than lower teeth (Fig. 1), with upper molars and first premolars, lower second molars and incisors more prone to loss, while as in other studies (e.g. Wood et al. 1989) the canines appear most resistant to breakdown leading to tooth loss. However, the annualized tooth loss rate in the Hirschfeld \& Wasserman (1978) study of maintained periodontal patients was at a smaller rate of 0.09 teeth per subject than the 0.25 teeth/patient/year in this present study. The annualized tooth loss rate in the present study was surprisingly comparable to that found in another university clinic population under regular maintenance of 0.23 teeth/patient/year (Tonetti et al. 1998), and better than that shown in one study of recall patients of 0.29 teeth/patient/year (Nabers et al. 1988), but as could be expected was more favorable than the rates of tooth loss in untreated periodontitis patients of 0.32 teeth/patient/year (Harris 2003) or 0.36 teeth/patient/year (Becker et al. 1979), and similar to but slightly less favorable than in a group of 44 patients treated but not maintained at 0.22 teeth/patient/year (Becker et al. 1984). 
Possible risk indicators associated with total tooth loss, tooth loss due to periodontal reasons, and current periodontal conditions of treated periodontal patients were explored in this study. Six factors, two of which could be modified namely smoking and use of interdental brush, were identified as being positively and negatively associated with total tooth loss and tooth loss due to periodontal reasons in the post-periodontal treatment period in the current study cohort (Tables $2 \& 3$ ). Age and time in years since periodontal therapy were also positively correlated with further tooth loss due to periodontal reasons. Education levels were negatively correlated with total tooth loss and periodontal tooth loss.

Age, smoking and education levels are among the well-established indicators and factors that have been reported to be related to loss of periodontal attachment (Ismail et al. 1990), with one recent study showing that in smokers other risk factors and indicators, such as education, are masked (Paulander et al. 2004). Older oriental SPC patients have been shown to be more likely to be compliant if targeted for clinicbased SPC recalls (Ojima et al. 2001) and so there may be justification in targeting older successfully treated periodontitis patients for clinic-based SPC recall. Tobacco smoking has been shown to adversely affect the outcome of scaling and root planing (Darby et al. 2005) and that of periodontal therapy over time (Ah et al. 1994, Boström et al. 1998), to be associated with increased subgingival calculus (Bergström 2005), to adversely affect tooth retention and/or periodontal parameters over the long-term (Krall et al. 1999, Bergstrom et al. 2000, Jansson \& Lavstedt 2002), and to exert a negative influence on outcomes of SPC and maintained oral hygiene (König 2002, Kerdvongbundit \& Wikesjo 2002). In one population of males with exposure to betel nut use however, tobacco use has not been shown to be a predictor of tooth loss over time (Neely et al. 2005). 
There could be little justification for committing the Periodontology Clinic's resources to trying to reduce the periodontal harm to which continuing smokers expose themselves. The only justification for targeting current smoking, treated periodontitis patients for clinic-based SPC would be the opportunity which regular SPC would offer for smoking cessation advice. Quitting smoking has been shown to have a beneficial effect with respect to tooth loss (Krall et al. 1997), bone loss (Bergström 2004) and to have a beneficial effect on reducing probing depths following non-surgical treatment (Preshaw et al. 2005). Thus positive good for tooth retention and periodontal status would eventuate from successful smoking cessation in smoking, treated periodontitis patients, shown to be at risk in this patient population. The role of the dental team in the promotion of smoking cessation has been emphasized (Newton \& Palmer 1997), however there is little evidence of the effectiveness of smoking cessation advice as part of SPC. One randomized controlled trial showed the effectiveness of hygienists in having smokeless tobacco using hygiene patients quit (Severson et al. 1998), and dental health care workers, as a group, have been shown to have promise in promoting quitting of tobacco use (Gordon \& Severson 2001).

Two-thirds of European dentists were shown to feel that smoking cessation is part of the dentist's duty, but barriers to acting on this were noted (Allard 2000). The effectiveness of tobacco cessation counseling in the dental office has been reviewed, and an expectation of a 10-15\% quit rate among tobacco using patients has been postulated (Warnakulasuriya 2002). Quit smoking strategies are being introduced to dental hygiene education and continuing education (Ramseier 2003, Monson \& Engeswick 2005) with some reported effectiveness. Training for the hygienists on smoking cessation strategies in the Periodontology Clinic which would offer SPC to 
treated periodontitis patients has commenced as a necessary prelude to the targeting of continuing smoking, periodontally treated periodontitis patients for clinic-based SPC recalls.

Regular SPC at three-monthly intervals is held out as making good for deficiencies in personal plaque control and poorer treatment responses (Ramfjord et al. 1982). The recolonization of subgingival niches by periodontopathogenic bacteria after subgingival debridement in the absence of good personal plaque control takes time (van Winkelhoff et al. 1988) and regular SPC is advocated to overcome deficiencies in oral hygiene (Ramfjord 1987). The patients in the present investigation had achieved good levels of plaque control, as evidenced by BOP levels at conclusion of periodontal therapy, and showed reasonably good treatment responses (Table 1). Targeting poor oral hygiene performers among treated periodontitis patients for regular clinic-based SPC would aim at establishing adequate levels of personal plaque control, which these patients demonstrated they could achieve during active periodontal therapy. While three-monthly (or three-to-fourmonthly) recalls are still advocated as appearing to be effective, subject to being varied depending upon patients' compliance and the dentist's clinical judgement (Cohen 2003), studies have demonstrated that the recall interval may be longer with no ill-effect for general dentistry patients (Rosen et al. 1999) and for hygiene patients (Johansson et al. 1984). The British National Institute for Clinical Excellence has recently promulgated a set of guidelines for dental recalls (National Institute for Clinical Excellence [NICE] 2004), reviewed by Bader (2005), which stress the individualized nature of the recall intervals and which also separate examination and intervention, both of which are inseparable according to the AAP position paper 
(Cohen 2003). Targeted clinic-based SPC recalls would be individualized and intervention would only follow establishment of a need.

Education level was negatively correlated with post-therapy further tooth loss due to all or periodontal reasons (Tables $2 \& 3$ ). The positive relationship of educational level and periodontal health in adults at a population level has been established for diverse populations (Ismail et al. 1990, Oliver et al. 1991, Locker \& Leake 1993, Gamonal et al. 1998, Katz et al. 2000, Corbet et al. 2001, Kocher et al. 2005), and education level has also been shown to be associated with tooth loss (Lin et al. 2001, Treasure et al. 2001). Targeting the less well educated for clinic-based SPC would involve a heavy emphasis on patient education about the nature of periodontal disease and its management and the role of self-care, in the hope that health education could to some extent impact upon the influence which may place such patients at a disadvantage. An analysis of education levels among the 97 cohorts vs their smoking habit revealed no significant association between the two (data not shown).

Self-reported time spent on daily oral hygiene was positively correlated with periodontal disease experience in terms of tooth loss. This apparently contradictory finding may be due to patients' awareness about existing on-going periodontal problems, which in turn probably resulted in more attention to daily oral hygiene. An alternative explanation might be that these subjects who had experienced progressive tooth loss had intentionally reported spending more time on their daily oral hygiene, while actually this was an exaggeration, based on a social desirability bias, which can commonly happen in response to questionnaires (Oppenheim 1992).

Self reported daily use of interdental brushes was negatively correlated with total tooth loss and tooth loss reported to be due to periodontal reasons (Tables $2 \& 3$ ). 
Kiger and co-workers (1991) have shown that interdental brush used in combination with a toothbrush is more effective in the removal of plaque from proximal tooth surfaces of treated periodontal patients than a toothbrush alone or toothbrush used in combination with dental floss. Use of multiple oral hygiene behaviours in addition to tooth brushing has been shown to lead to decreased tooth loss (Kressin et al. 2003). There is some dispute as to the part played by subgingival scaling during SPC (Jenkins et al. 2000). However, the premise that supragingival plaque control is an important and integral element of maintaining periodontal health (Corbet \& Davies 1993) has been reaffirmed in the systematic review of SPC (Heasman et al. 2002). It is logical to expect that those individuals who employed more effective oral hygiene measures (e.g. interdental brushes) would experience less periodontal disease complications such as tooth loss, despite the lack of SPC delivered in the Periodontology Clinic. Periodontitis patients who fail to master appropriate interdental oral hygiene measures could be another group targeted for clinic-based SPC until mastery of interdental cleaning is achieved, although poorly performing treated periodontitis patients may have low-compliance (Ojima et al. 2005).

When analyzed independently by arch, wearing of upper or lower removable partial dentures was related to tooth loss in the same arch due to all and periodontal reasons. The impact of removable partial denture wearing on periodontal health has regularly been reported to be unfavorable (Carlsson et al. 1976, Markkanen et al. 1987, Yusof \& Isa 1994, Corbet et al. 2001), although some studies show no such unfavorable periodontal outcomes (Chandler \& Brudvik 1984). Better oral hygiene is generally associated with less detrimental periodontal effects of removable partial denture wearing (Carlsson et al. 1976) and regular recall for removable partial denture wearing patients has mostly been shown to reduce the periodontal consequences 
(Drake \& Beck 1993), although one study from a country with no access to dental hygienists and in a population with relatively low level of oral hygiene, six monthly recalls had no beneficial effects (Vanzeveren et al. 2002). It seems reasonable therefore to select treated periodontitis patients rehabilitated by means of removable partial dentures for regular recall within the Periodontology Clinic.

This group of treated periodontitis patients who had shown initially reasonable good treatment responses but who had sought less than optimal supportive periodontal therapy, showed deterioration in their periodontal condition over time. Tobacco smoking was an important risk factor for periodontal deterioration in this group and as such tobacco cessation should be part of SPC efforts for treated periodontitis patients who continue smoke. The subjects' risk to tooth loss seems to be reduced if they adopted more effective plaque control measures. Continuing smokers, older treated periodontitis patients and patients with low educational level, as identified in this study, should be those targeted for clinic-based SPC in this teaching Periodontology Clinic.

\section{Acknowledgement}

The work described in this paper was fully supported by a grant from the Research Grants Council of the Hong Kong Special Administrative Region, China (HKU 7331/00M). 


\section{References}

Ah, M.K., Johnson, G.K., Kaldahl, W.B., Patil, K.D. \& Kalkwarf, K.L. (1994) The effect of smoking on the response to periodontal therapy. Journal of Clinical Periodontology 21, 91-97.

Ainamo, J. \& Ainamo, A. (1996) Risk assessment of recurrence of disease during supportive periodontal care. Epidemiological considerations. Journal of Clinical Periodontology 23, 232-239.

Allard, R.H. (2000) Tobacco and oral health: attitudes and opinions of European dentists: a report of the EU working group on tobacco and oral health. International Dental Journal 50, 99-102.

American Academy of Periodontology (1998) Position paper. Supportive periodontal therapy. Journal of Periodontology 69, 502-506.

Anneken, C., Treinen, J. \& Willershausen, B. (2001) Patients compliance in periodontal therapy: a retrospective investigation on the basis of a clinical group. European Journal of Medical Research. 28, 75-82.

Antczak-Bouckoms, A. (1994) Natural history of periodontitis and a review of technologies to prevent and treat it. Journal of Dental Education 58, 625640.

Axelsson, P. \& Lindhe, J. (1981) Effect of controlled oral hygiene procedures on caries and periodontal disease in adults. Results after 6 years. Journal of Clinical Periodontology 8, 239-248.

Axelssson, P., Nyström B. \& Lindhe, J. (2004) The long-term effect of a plaque control program on tooth mortality, caries and periodontal disease in adults. Results after 30 years of maintenance. Journal of Clinical Periodontology 31, 749-757. 
Axtelius, B, Soderfeldt, B., Edwardsson, S. \& Attstrom, R. (1997) Therapy-resistant periodontitis (I). Clincal and treatment characteristics. Journal of Clinical Periodontology 24, 640-645.

Bader, J. (2005) Risk-based recall intervals recommended. Evidence-Based Dentistry 6, 2-4.

Baelum, V., Luan, W.M., Chen, X. \& Fejerskov, O. (1997) Predictors of tooth loss over 10 years in adult and elderly Chinese. Community Dentistry and Oral Epidemiology 25, 204-210.

Beck, J.D. (1994) Methods of assessing risk for periodontitis and developing multifactorial models. Journal of Periodontology 65, 468-478.

Becker, W., Becker, B.E. \& Berg, L.E. (1984) Periodontal treatment without maintenance. A retrospective study in 44 patients. Journal of Periodontology 55, 505-509.

Becker, W., Berg, L.E. \& Becker, B.E. (1979) Untreated periodontal disease: a longitudinal study. Journal of Periodontology 50, 234-244.

Bergström, J. (2004) Influence of tobacco smoking on periodontal bone height. Longterm observations and a hypothesis. Journal of Clinical Periodontology 31, 260-266.

Bergström, J. (2005) Tobacco smoking and subgingival dental calculus. Journal of Clinical Periodontology 32, 81-88.

Bergström, J., Eliasson, S. \& Dock, J. (2000) A 10-year prospective study of tobacco smoking and periodontal health. Journal of Periodontology 71, 1338-1347.

Boström, L., Linder, L.E. \& Bergstrom, J. (1998) Influence of smoking on the outcome of periodontal surgery. A 5-year follow-up. Journal of Clinical Periodontology 25, 194-201. 
Carlsson, G.E., Hedegard, B. \& Koivumaa, K.K. (1976) Late results of treatment with partial dentures. An investigation by questionnaire and clinical examination 13 years after treatment. Journal of Oral Rehabilitation 3, 267-272.

Census and Statistics Department, Hong Kong (1992) Hong Kong 1991 Population Census. Hong Kong: Hong Kong Census and Statistics Department.

Chandler, J.A. \& Brudvik, J.S. (1984) Clinical evaluation of patients eight to nine years after placement of removable partial dentures. Journal of Prosthetic Dentistry 51, 736-743.

Checchi, L., Montevecchi, M., Gatto, M.R. \& Trombelli, L. (2002) Retrospective study of tooth loss in 92 treated periodontal patients. Journal of Clinical Periodontology 29, 651-656.

Checchi, L., Pelliccioni, G,A., Gatto, M.R. \& Kelescian, L. (1994) Patient compliance with maintenance therapy in an Italian periodontal practice. Journal of Clinical Periodontology 21, 309-312.

Claffey, N. \& Egelberg, J. (1995) Clinical indicators of probing attachment loss following initial periodontal treatment in advanced periodontitis patients. Journal of Clinical Periodontology 22, 690-696.

Cohen, R.E. (2003) Position paper : periodontal maintenance. American Academy of Periodontology Journal of Periodontology 74,1395-1401.

Corbet, E.F. \& Davies, W.I.R. (1991) Reasons given for tooth extraction in Hong Kong. Community Dental Health 8, 121-130.

Corbet, E.F. \& Davies, W.I.R. (1993). The role of supragingival plaque in the control of progression periodontal disease. A review. Journal of Clinical Periodontology 20, 307-313. 
Corbet, E.F., Wong, M.C.M. \& Lin, H.C. (2001) Periodontal conditions in adult Southern Chinese. Journal of Dental Research 80, 1480-1485.

Darby, I.B., Hodge, P.J., Riggio, M.P. \& Kinane, D.F. (2005) Clinical and microbiological effect of scaling and root planing in smoker and non-smoker chronic and aggressive periodontitis patients. Journal of Clinical Periodontology 32, 200-206.

Demetriou, N., Tasmi-Pandi, A. \& Parashis, A. (1995) Compliance with supportive periodontal treatment in private periodontal practice. A 14-year retrospective study. Journal of Periodontology 66, 145-149.

Drake, C.W. \& Beck, J.D. (1993) The oral status of elderly removable partial denture wearers. Journal of Oral Rehabilitation 20, 53-60.

Fardal, Ø., Johannessen, A.C. \& Linden, G.J. (2003) Compliance in a Norwegian periodontal practice. Oral Health \& Preventive Dentistry 1, 93-98.

Flemmig, T.F., Weinacht, S., Rüdiger, S., Rumetsch, M., Jung, A. \& Klaiber, B. (1996) Adjunctive controlled topical application of tetracycline $\mathrm{HCl}$ in the treatment of localized persistent or recurrent periodontitis. Effects on clinical parameters and elastase- $\alpha_{1}$-proteinase inhibitor in gingival crevicular fluid. Journal of Clinical Periodontology 23, 914-921.

Gamonal, J.A., Lopez, N.J. \& Aranda, W. (1998) Periodontal conditions and treatment needs, by CPITN, in the 35-44 and 65-74 year-old population in Santiago, Chile. International Dental Journal 48, 96-103.

Genco, R.J., Ho, A.W., Grossi, S.G., Dunford, R.G., Tedesco, L.A. (1999) Relationship of stress distress and inadequate coping behaviors to periodontal disease. Journal of Periodontology 70, 711-723. 
Gilbert, G.H., Shelton, B.J., Chavers, L.S. \& Bradford, E.H. Jr. (2002) Predicting tooth loss during a population-based study: role of attachment level in the presence of other dental conditions. Journal of Periodontology 73, 14271436.

Goldman, M.C., Ross, I.F. \& Goteiner, D. (1986) Effect of periodontal therapy on patients maintained for 15 years or longer. A retrospective study. Journal of Periodontology 57, 347-353.

Gordon, J.S. \& Severson, H.H. (2001) Tobacco cessation through dental office settings. Journal of Dental Education 65, 354-363.

Harris, R.J. (2003) Untreated periodontal disease: a follow-up on 30 cases. Journal of Periodontology 74, 672-678.

Heasman, P.A., McCracken, G.I. \& Steen, N. (2002) Supportive periodontal care: the effect of periodic subgingival debridement compared with supragingival prophylaxis with respect to clinical outcomes. Journal of Clinical Periodontology 29 (Suppl 3), 163-172.

Heitz-Mayfield, L.J.A. (2005) Disease progression: identification of high-risk groups and individuals for periodontitis. Journal of Clinical Periodontology 32 (Suppl 6), 196-209.

Heitz-Mayfield, L.J.A., Trombelli, L., Heitz, F., Needleman, I. \& Moles, D. (2002) A systematic review of the effect of surgical debridement vs non-surgical debridement for the treatment of chronic periodontitis. Journal of Clinical Periodontology 29 (Suppl 3.), 92-102.

Hirschfeld, L. \& Wasserman, B. (1978) A long-term survey of tooth loss in 600 treated periodontal patients. Journal of Periodontology 49, 225-237. 
Ismail, A.I., Morrison, E.C., Burt, B.A., Caffesse, R.G. \& Kavanagh, M.T. (1990) Natural history of periodontal disease in adults: Findings from the Tecumseh periodontal disease study, 1959-87. Journal of Dental Research 69, 430435.

Jansson, L.E. \& Hagstrom K.E. (2002) Relationship between compliance and periodontal treatment outcome in smokers. Journal of Periodontology 73, 602-607.

Jansson, L.E. \& Lavstedt, S. (2002) Influence of smoking on marginal bone loss and tooth loss - a prospective study over 20 years. Journal of Clinical Periodontology 29, 750-756.

Jenkins W.M., Said S.H., Radvar M. \& Kinane D.F. (2000) Effect of subgingival scaling during supportive therapy. Journal of Clinical Periodontology 27, 590-596.

Johansson, L.A., Oster, B. \& Hamp, S.E. (1984) Evaluation of cause-related periodontal therapy and compliance with maintenance care recommendations. Journal of Clinical Periodontology 11, 689-699.

Joss, A., Adler, R. \& Lang, N.P. (1994) Bleeding on probing. A parameter for monitoring periodontal conditions in clinical practice. Journal of Clinical Periodontology 21,402-408.

Katz, J., Peretz, B., Sgan-Cohen, H.D., Horev, T. \& Eldad, A. (2000) Periodontal status by CPITN, and associated variables in an Israeli permanent force military population. Journal of Clinical Periodontology 27, 319-324.

Kerdvongbundit, V. \& Wikesjo, U.M. (2002) Prevalence and severity of periodontal disease at mandibular molar teeth in smokers with regular oral hygiene habits. Journal of Periodontology 73, 735-740. 
Kiger, R.D., Nyland, K. \& Feller, R.P. (1991) A comparison of proximal plaque removal using flossing and interdental brushes. Journal of Clinical Periodontology 18, 681-684.

Kocher, T., Schwahn, C., Gesch, D., Bernhardt, O., John, U., Meisel, P. \& Baelum, V. (2005) Risk determinants of periodontal disease - an analysis of the Study of Health in Pomerania (SHIP 0) Journal of Clinical Periodontology 32, 59-67.

König, J., Plagmann, H.C., Ruhling, A. \& Kocher, T. (2002) Tooth loss and pocket probing depths in compliant periodontally treated patients: a retrospective analysis. Journal of Clinical Periodontology 29, 1092-1100.

Kornman, K.S., Page, R.C. \& Tonetti, M.S. (1997) The host response to the microbiol challenge in periodontitis; assembling the players. Periodontology 2000 14, 33-53.

Krall, E.A., Dawson-Hughes, B., Garvey, A.J. \& Garcia, R.I. (1997) Smoking, smoking cessation, and tooth loss. Journal of Dental Research 76, 16531659.

Krall, E.A., Garvey, A.J. \& Garcia, R.I. (1999) Alveolar bone loss and tooth loss in male cigar and pipe smokers. Journal of the American Dental Association 130, $57-64$.

Kressin, N.R., Boehmer, U., Nunn, M.E. \& Spiro, A. 3rd. (2003) Increased preventive practices lead to greater tooth retention. Journal of Dental Research 82, 223227.

Lin, H.C., Corbet, E.F., Lo, E.C. \& Zhang, H.G. (2001) Tooth loss, occluding pairs, and prosthetic status of Chinese adults. Journal of Dental Research 80, 14911495. 
Locker, D. \& Leake, J.L. (1993) Risk indicators and risk markers for periodontal disease in older adults living independently in Ontario, Canada. Journal of Dental Research 72, 9-17.

Markkanen, H., Lappalainen, R., Honkala, E. \& Tuominen, R. (1987) Periodontal conditions with removable complete and partial dentures in the adult population aged 30 years and over. Journal of Oral Rehabilitation 14, 355360.

McFall, W.T. (1982) Tooth loss in 100 treated patients with periodontal disease. Journal of Periodontology 53, 539-549.

McLeod, D.E., Lainson, P.A. \& Spivey, J.D. (1997) The effectiveness of periodontal treatment as measured by tooth loss. Journal of the American Dental Association 128, 316-324.

Mendoza, A.R., Newcomb, G.M. \& Nixon, K.C. (1991) Compliance with supportive periodontal therapy. Journal of Periodontology 62, 731-736.

Molloy, J. Wolff, L.F., Lopez-Guzman, A. \& Hodges, J.S. (2004) The association of periodontal disease parameters with systemic medical conditions and tobacco us. Journal of Clinical Periodontology 31, 625-632.

Monson, A.L. \& Engeswick, L.M. (2005) Promotion of tobacco cessation through dental hygiene education: a pilot study. Journal of Dental Education 69, 901911.

Nabers, C.L., Stalker, W.H., Esparza, D., Naylor, B. \& Canales, S. (1988) Tooth loss in 1535 treated periodontal patients. Journal of Periodontology 59, 297-300.

National Institute for Clinical Excellence (2004) Clinical Guideline 19. Dental recall: recall interval between routine dental examinations. [WWW document] 
URL http:// www.nice.org.uk/CG019NICEguideline [accessed on 14 September 2005]

Neely, A.L., Holford, T.R., Löe, H, Ånerud, Å. \& Boysen, H. (2005) The natural history of periodontal disease in humans: risk factors for tooth loss in cariesfree subjects receiving no oral health care. Journal of Clinical Periodontology 32, 984-993.

Newton J.T. \& Palmer R.M. (1997) The role of the dental team in the promotion of smoking cessation. British Dental Journal 10, 353-355.

Ojima, M., Hanioka, T. \& Shizukuishi, S. (2001) Survival analysis for degree of compliance with supportive periodontal therapy. Journal of Clinical Periodontology 28, 1091-1095.

Ojima, M., Kanagawa, H., Nishida, N., Hanioka, T, \& Shizukuishi, S. (2005) Relationship between attitudes toward oral health at initial office visit and compliance with supportive periodontal treatment. Journal of Clinical Periodontology 32, 364-368.

Oliver, R.C., Brown, L.J. \& Loe, H. (1991) Variations in the prevalence and extent of periodontitis. Journal of the American Dental Association 122, 43-48.

Oppenheim, A.N. (1992) Questionnaire design, interviewing and attitude measurement. London: Pinter Publishers.

Paulander, J., Wennström, J.L., Axelsson, P. \& Lindhe, J. (2004) Some risk factors for periodontal bone loss in 50-year-old individuals. Journal of Clinical Periodontology 31, 489-496.

Persson, R.E., Tzannetou, S., Feloutzis, A.G., Bragger, U., Persson, G.R., \& Lang, N.P. (2003) Comparison between panoramic and intra-oral radiographs for 
the assessment of alveolar bone levels in a periodontal maintenance population. Journal of Clinical Periodontology 30, 833-839.

Preshaw, P.M. \& Heasman, P.A. (2005) Periodontal maintenance in a specialist periodontal practice and in general dental practice. Journal of Clinical Periodontology 32, 280-286.

Preshaw, P.M., Heasman, L., Stacey, F., Steen, N., McCracken, G.I., \& Heasman, P.A. (2005) The effect of quitting smoking on chronic periodontitis. Journal of Clinical Periodontology 32, 869-879.

Rahardjo, A., Yoshihara, A., Amarasena, N., Ogawa, H., Nakashima K. \& Miyazaki, H. (2005) Relationship between bleeding on probing and periodontal disease progression in community-dwelling older adults. Journal of Clinical Periodontology 32, 1129-1133.

Ramfjord, S.P. (1987) Maintenance care for treated periodontitis patients. Journal of Clinical Periodontology 14, 433-437.

Ramfjord, S.P., Morrison, E.C., Burgett, F.G., Nissle, R.R., Shick, R.A., Zann, G.J. \& Knowles, J.W. (1982) Oral hygiene and maintenance of periodontal support. Journal of Periodontology 53, 26-30.

Rams, T.E., Listgarten, M.A. \& Slots, J. (1996) Utility of 5 major putative periodontal pathogens and selected clinical parameters to predict periodontal breakdown in patients on maintenance care. Journal of Clinical Periodontology 23, 346-354.

Ramseier, C.A. (2003) Smoking prevention and cessation. Oral Health and Preventive Dentistry 1 (Suppl 1), 427-439.

Renvert, S. \& Persson, G.R. (2002) A systematic review on the use of residual probing depth, bleeding on probing and furcation status following initial 
periodontal therapy to predict further attachment and tooth loss. Journal of Clinical Periodontology 29 (Suppl. 3), 82-89.

Rosen, B., Olavi, G., Badersten, A., Ronstrom, A., Soderholm, G. \& Egelberg, J. (1999) Effect of different frequencies of preventive maintenance treatment on periodontal conditions. 5-Year observations in general dentistry patients. Journal of Clinical Periodontology 26, 225-233.

Rosling, B., Serino, G., Hellstrom, M.K., Socransky, S.S. \& Lindhe, J. (2001) Longitudinal periodontal tissue alterations during supportive therapy. Findings from subjects with normal and high susceptibility to periodontal disease. Journal of Clinical Periodontology 28, 241-249.

Schätzle, M., Löe, H., Lang, N.P., Bürgin, W., Ánerud, Á. \& Boysen, H. (2004) The clinical course of chronic periodontitis. Journal of Clinical Periodontology 31, 1122-1127.

Severson, H.H., Andrews, J.A., Lichtenstein, E., Gordon, J.S. \& Barckley, M.F. (1998) Using the hygiene visit to deliver a tobacco cessation program: results of a randomized clinical trial. Journal of American Dental Association 129, 993 999.

Soolari, A. \& Rokn, A.R. (2003) Adherence to periodontal maintenance in Tehran, Iran. A 7-year retrospective study. Quintessence International 34, 215-219.

Tonetti, M.S., Muller-Campanile, V. \& Lang N.P. (1998) Changes in the prevalence of residual pockets and tooth loss in treated periodontal patients during a supportive maintenance care program. Journal of Clinical Periodontology 25, 1008-1016.

Tonetti, M.S., Steffen, P., Muller-Campanile, V., Suvan, J. \& Lang, N.P. (2000) Initial extractions and tooth loss during supportive care in a periodontal 
population seeking comprehensive care. Journal of Clinical Periodontology 27, 824-831.

Treasure, E., Kelly, M., Nuttall, N., Bradnock, G. \& White, D. (2001) Factors associated with oral health: a multivariate analysis of results from the 1998 Adult Dental Health survey. British Dental Journal 190, 60-68.

van der Weijden, G.A. \& Timmerman, M.F. (2002) A systematic review on the clinical efficacy of subgingival debridement in the treatment of chronic periodontitis. Journal of Clinical Periodontology 29 (Suppl. 3), 55-71.

van Winkelhoff, A.J., van der Velden, U. \& de Graaff, J. (1988) Microbial succession in recolonizing deep periodontal pockets after a single course of supra- and subgingival debridement. Journal of Clinical Periodontology 15, 116-22.

Vanzeveren, C., D'Hoore, W. \& Bercy, P. (2002) Influence of removable partial denture on periodontal indices and microbiological status. Journal of Oral Rehabilitation 29, 232-239.

Warnakulasuriya, S. (2002) Effectiveness of tobacco counselling in the dental office. Journal of Dental Education 66, 1079-1087.

Wilson, T.G., Glover, M.E., Schoen, J., Baus, C. \& Jacobs T. (1984) Compliance with maintenance therapy in a private practice. Journal of Periodontology 55, 468-473.

Wilson, T.G., Hale, S. \& Temple, R. (1993) The results of efforts to improve compliance with supportive treatment in a private practice. Journal of Periodontology 64, 311-314.

Wood, W.R., Greco, G.W. \& McFall, W.T. (1989) Tooth loss in patients with moderate periodontitis after treatment and long-term maintenance care. Journal of Periodontology 60, 516-520. 
Worthington, H., Clarkson, J. \& Davies, R. (1999) Extraction of teeth over 5 years in regularly attending adults. Community Dentistry and Oral Epidemiology 27, 187-194.

Yusof, Z. \& Isa, Z. (1994) Periodontal status of teeth in contact with denture in removable partial denture wearers. Journal of Oral Rehabilitation 21, 77-86.

Address:

W. Keung Leung

Faculty of Dentistry

The University of Hong Kong

Room 3B39, Prince Philip Dental Hospital, 34 Hospital Road

Hong Kong SAR

China

Tel: $\quad 852-2859-0417$

Fax: $\quad 852-2858-7874$

Email: ewkleung@hkucc.hku.hk 


\section{Legend}

Fig. 1. Count of individual tooth remaining after previous periodontal therapy; number of total tooth loss, and self-reported tooth loss due to periodontal reasons at re-examination. A marked bilateral symmetry of teeth remaining as well as tooth loss was noted and hence left and right teeth are grouped together. Black squares: number of teeth remaining after previous periodontal therapy; black circles: number of total tooth lost; open circles: number of self-reported tooth lost due to periodontal reasons.

Fig. 2. Percentage of tooth lost over the post-treatment period. Black columns: percentage of total tooth lost; white columns: self-reported percentage of tooth lost due to periodontal reasons.

Fig. 3. Percentage of subjects by number of teeth lost. Black columns: teeth lost due to all reasons; white columns: self-reported teeth lost due to periodontal reasons. 


\section{Clinical Relevance}

Scientific Rationale: Staffing, logistic arrangements and lack of compliance often mean that not all treated periodontitis patients benefit from clinic-based supportive periodontal care (SPC). Principal Findings: At recall after 5-12 years in periodontitis patients who were successfully treated but discharged with advice on SPC, smokers, removable partial denture wearers, more elderly patients, patients with lower education levels or not using interdental brushes experienced more tooth loss.

Practical Implications: In circumstances in which not all treated periodontitis patients can be enrolled in clinic-based SPC programmes this study suggests patient categories deserving of enrolment in clinic-based SPC. 
Table 1. Range, mean ( \pm standard deviation) full-month clinical parameters of participants

\begin{tabular}{|c|c|c|c|}
\hline & $\begin{array}{l}\text { Before previous } \\
\text { periodontal } \\
\text { therapy* } \\
(\mathrm{n}=97)\end{array}$ & $\begin{array}{l}\text { conclusion of } \\
\text { previous } \\
\text { periodontal } \\
\text { therapy* } \\
(\mathrm{n}=97)\end{array}$ & $\begin{array}{l}\text { Re-examination }^{\dagger} \\
\quad(\mathrm{n}=96)\end{array}$ \\
\hline Plaque $\%$ & ND & ND & $\begin{array}{c}7.4-100 \\
(68.6 \pm 23.4)\end{array}$ \\
\hline BOP \% & $\begin{array}{c}42.0-91.7 \\
(71.3 \pm 17.7)\end{array}$ & $\begin{array}{c}0-20.2 \\
(5.5 \pm 5.5)\end{array}$ & $\begin{array}{c}11.8-94.7 \\
(46.7 \pm 19.3)\end{array}$ \\
\hline$\%$ sites with $\mathrm{PPD} \geq 6 \mathrm{~mm}$ & $\begin{array}{c}3.3-92.3 \\
(25.5 \pm 16.4)\end{array}$ & 0 & $\begin{array}{c}0-26.8 \\
(3.1 \pm 4.6)\end{array}$ \\
\hline Number of teeth & $\begin{array}{c}14-32 \\
(27.1 \pm 3.6)\end{array}$ & $\begin{array}{c}13-32 \\
(26.1 \pm 4.1)\end{array}$ & $\begin{array}{c}0-32^{\ddagger} \\
(23.3 \pm 5.6)\end{array}$ \\
\hline $\begin{array}{l}{ }^{*} \text { manual probing measurer } \\
{ }^{\dagger} \text { measurement by Florida } \mathrm{F} \\
{ }^{\dagger} \mathrm{n}=97 .\end{array}$ & $\begin{array}{l}\text { at from participan } \\
\mathrm{be}^{\circledR} \text {; one subject }\end{array}$ & $\begin{array}{l}\text { clinical records. } \\
\text { tulous. }\end{array}$ & \\
\hline
\end{tabular}


Table 2. Linear multiple regression model of further tooth loss due to all reasons among the participants*

\begin{tabular}{|c|c|c|c|}
\hline Variable & $\beta$ & $\operatorname{SE~} \beta$ & $p$-value \\
\hline Smoking-pack-years & 0.101 & 0.024 & $<0.0001$ \\
\hline Self-reported daily oral hygiene duration & 0.188 & 0.055 & 0.001 \\
\hline Years elapsed since treatment & 0.367 & 0.109 & 0.0011 \\
\hline Age & 0.088 & 0.027 & 0.0014 \\
\hline Interdental brush usage & -1.138 & 0.498 & 0.0247 \\
\hline Education level & -0.438 & 0.192 & 0.0249 \\
\hline (Constant) & -4.615 & 1.692 & 0.0077 \\
\hline
\end{tabular}


Table 3. Linear multiple regression model of further tooth loss due to periodontal reasons among the participants*

\begin{tabular}{lccc}
\hline \multicolumn{1}{c}{ Variable } & $\beta$ & SE $\beta$ & $p$-value \\
\hline Smoking-pack-years & 0.110 & 0.023 & $<0.0001$ \\
Self-reported daily oral hygiene duration & 0.193 & 0.053 & 0.0004 \\
Years elapsed since treatment & 0.347 & 0.104 & 0.0013 \\
Age & 0.065 & 0.026 & 0.0127 \\
Interdental brush usage & -1.114 & 0.478 & 0.0221 \\
$\begin{array}{l}\text { Education level } \\
\text { (Constant) }\end{array}$ & -0.377 & 0.184 & 0.0441 \\
\hline *Adjusted $R^{2}=0.400, F=11.675$, & $p<0.0001$. & Analysis performed as per \\
recommendations by Beck $(1994)$. & -4.180 & 1.625 & 0.0117 \\
\end{tabular}




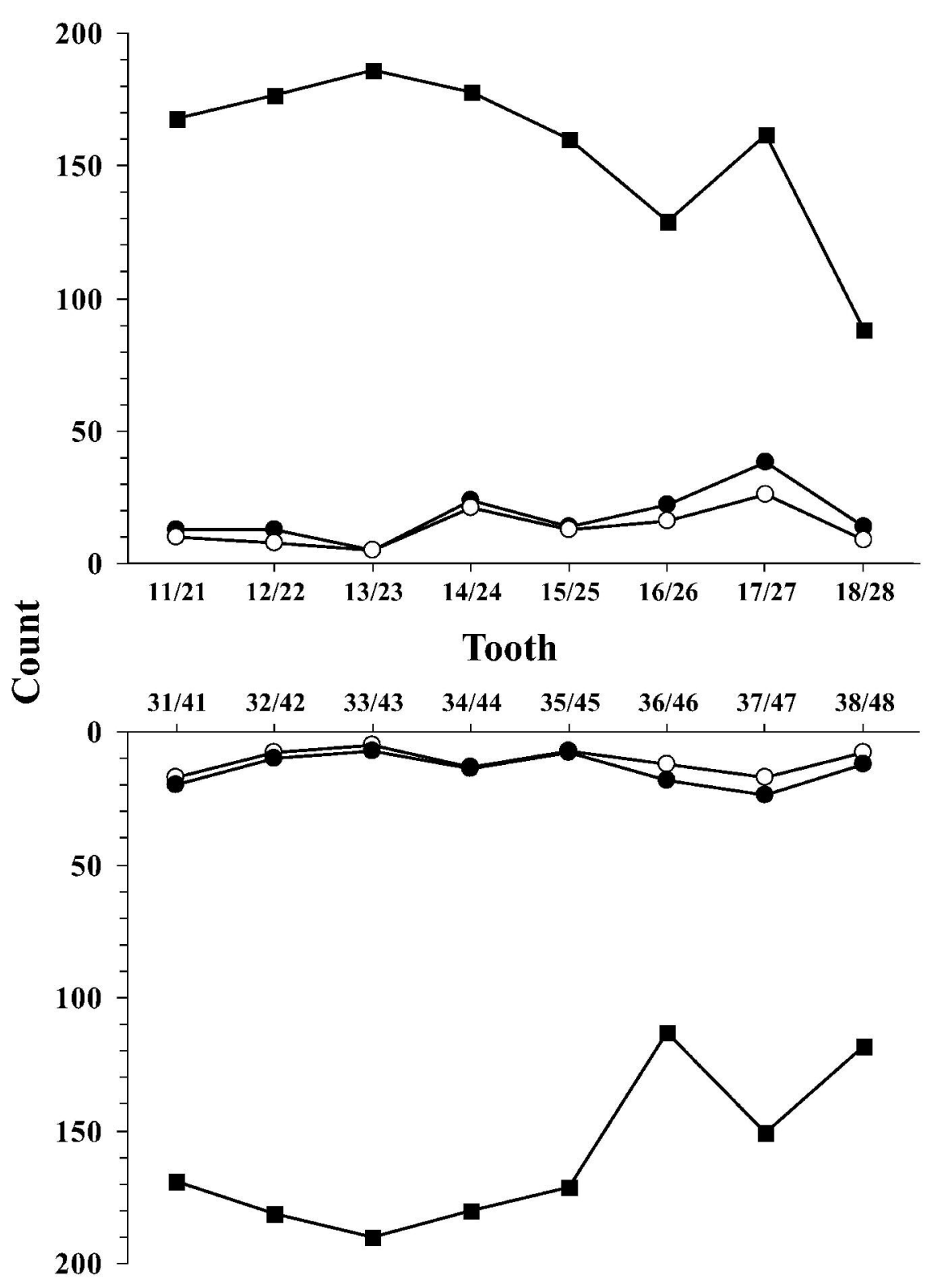

Journal of Clinical Periodontology - PROOF 


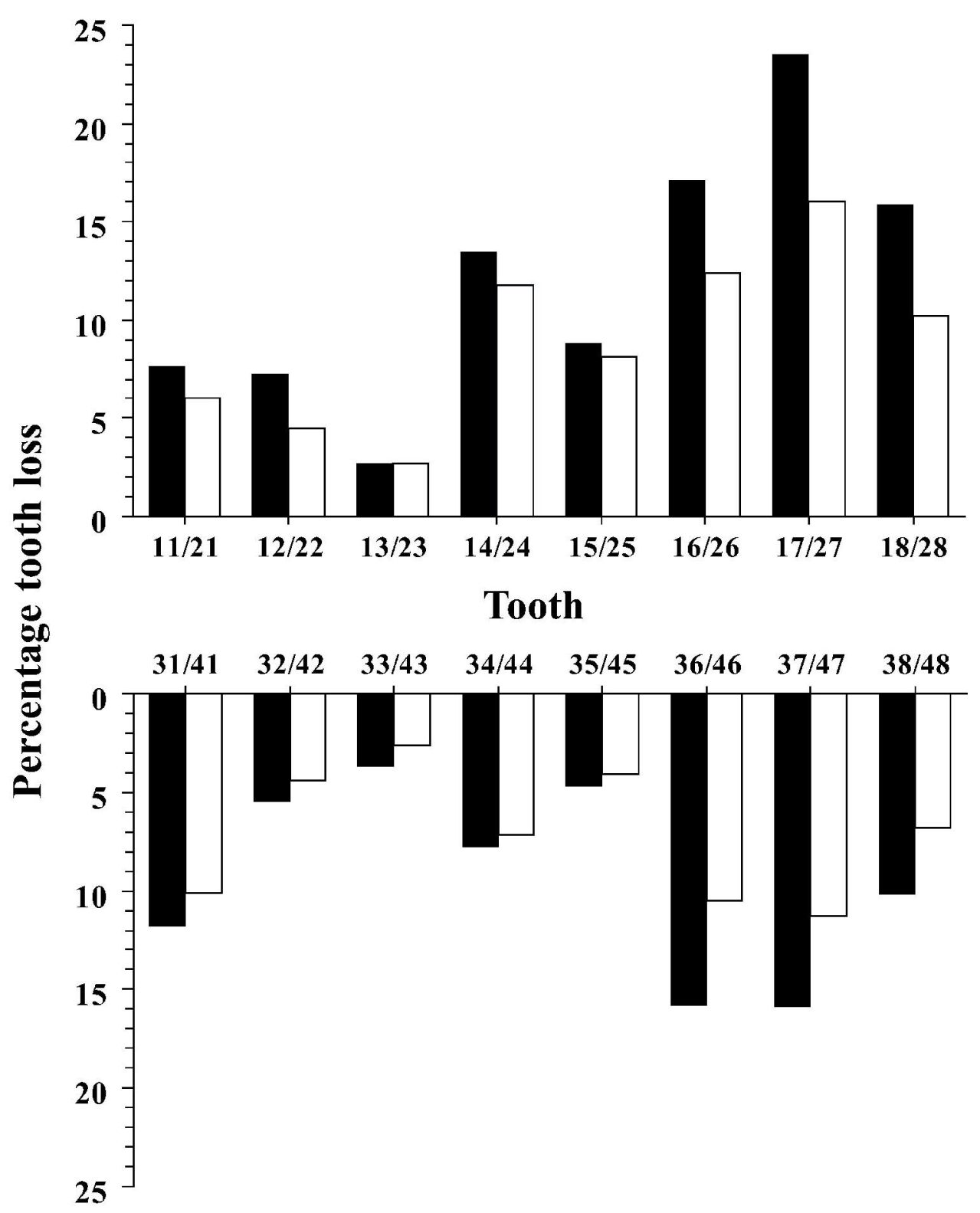

Journal of Clinical Periodontology - PROOF 


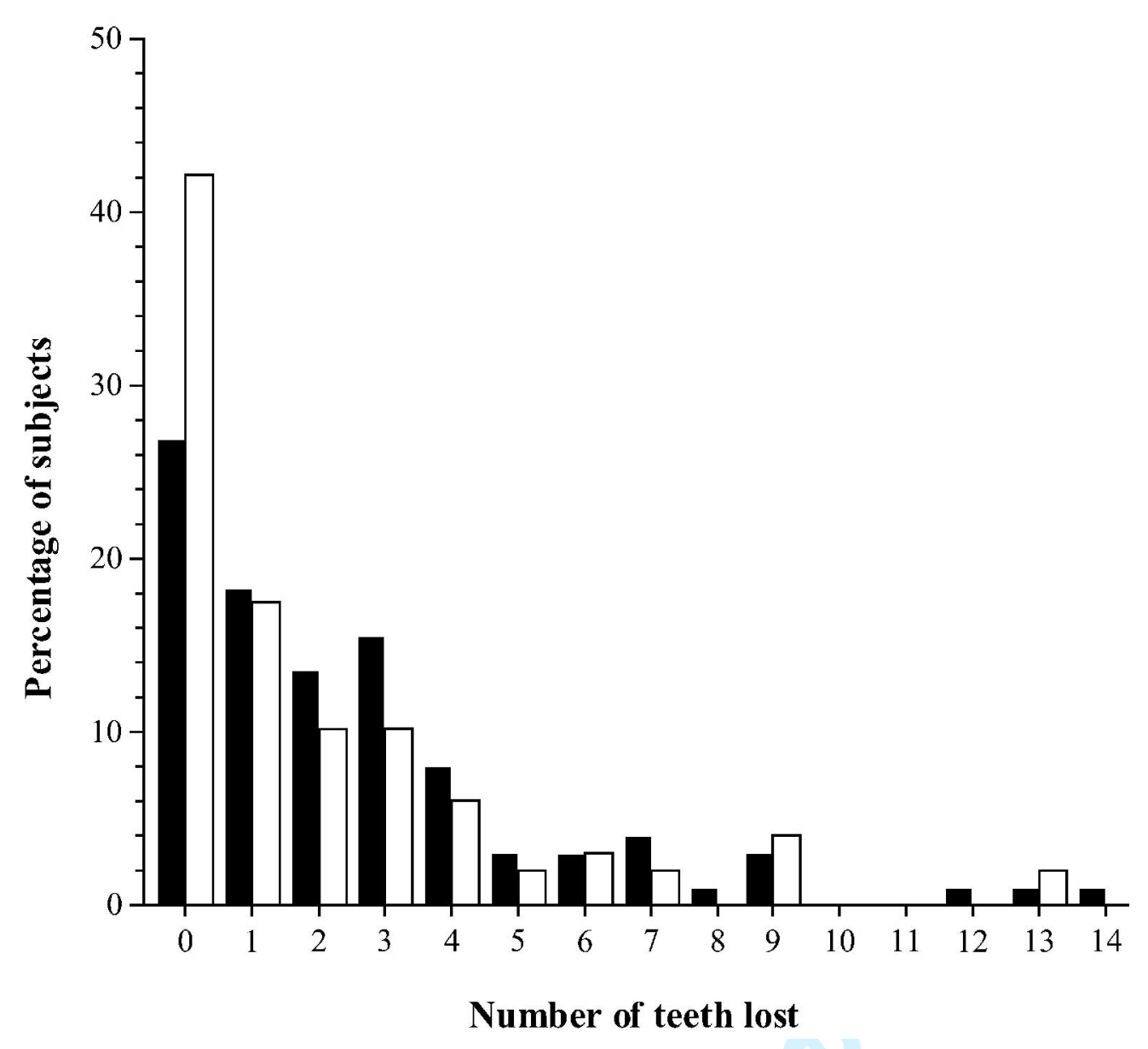

Journal of Clinical Periodontology - PROOF 